\title{
Communication
}

\section{The Single Nuclear Lamin of Caenorhabditis elegans Forms In Vitro Stable Intermediate Filaments and Paracrystals with a Reduced Axial Periodicity}

\author{
Anton Karabinos ${ }^{1}$, Jürgen Schünemann ${ }^{1}$, Michael Meyer ${ }^{1}$, Ueli Aebi ${ }^{2}$ \\ and Klaus Weber ${ }^{1 *}$
}

\author{
${ }^{1}$ Max Planck Institute for \\ Biophysical Chemistry \\ Department of Biochemistry \\ Am Fassberg 11, 37077 \\ Göttingen, Germany
}

${ }^{2}$ M. E. Müller-Institute for Microscopy at the Biozentrum University of Basel

Klingelbergstrasse 70, CH-4056

Basel, Switzerland

\begin{abstract}
The lamins of the tunicate Ciona intestinalis and the nematode Caenorhabditis elegans show unusual sequence features when compared to the more than 35 metazoan lamin sequences currently known. We therefore analyzed the in vitro assembly of these two lamins by electron microscopy using chicken lamin B2 as a control. While lamin dimers usually appear as a rod carrying two globules at one end, these globules are absent from Ciona lamin, which lacks the central 105-residue region of the tail domain. The deletion of 14 residues or two heptads from the coiled coil rod domain of the single C. elegans lamin results in a 1.5-nm shortening of the dimer rod. Similarly, the paracrystals assembled from the C. elegans lamin exhibit a 3.1-nm reduction of the true axial repeat compared to that of chicken lamin B2 paracrystals. We speculate that the banding pattern in the C. elegans lamin paracrystals arises from a relative stagger between dimers and/or a positioning of the globular tail domain relative to the central rod that is distinct from that observed in chicken lamin B2 paracrystals. Here we show that a nuclear lamin can assemble in vitro into $10-\mathrm{nm}$ intermediate filaments (IFs). C. elegans lamin in low ionic strength Tris-buffers at a $\mathrm{pH}$ of 7.2-7.4 provides a stable population of lamin IFs. Some implications of this filament formation are discussed.
\end{abstract}

(C) 2003 Elsevier Science Ltd. All rights reserved

Keywords: Ciona intestinalis; Caenorhabditis elegans; intermediate filament; lamin; paracrystals rod carrying two globular domains at one end. Thus, the two-stranded coiled coil is formed by parallel $\alpha$-helices without any stagger. The two globules represent the large lamin tail domains, since they are absent in the tailless lamin constructs but are retained in the headless variants. In the next assembly step, lamin dimers form polar head-to-tail polymers in which the highly conserved C-terminal end segment of one rod domain overlaps the highly conserved N-terminal end segment of the next rod domain. The formation of these very thin polar filaments requires the presence of at least the C-terminal part of the small head domain. Rather than forming stable $10-n m$ IF the next step of lamin polymerization leads to thick filament bundles, tangles and paracrystalline arrays. ${ }^{1,2,6,7}$ Thus, in contrast to cytoplasmic IF proteins 10-nm-like filaments are only transiently observed during lamin assembly and are practically absent at steady state. ${ }^{6,8}$

\begin{abstract}
Abbreviation used: IF, intermediate filament.
E-mail address of the corresponding author: akarabi1@gwdg.de lamins. ${ }^{2,3}$

The nuclear lamins have the tripartite domain (IF) proteins. A central $\alpha$-helical rod domain to form a segmented double-stranded coiled coil, is flanked by $\mathrm{N}$ and C-terminal head and tail domains. ${ }^{4,5}$ Electron microscopy of various lamins and lamin-derived constructs has defined the in vitro assembly pathway. The dimer is a $52 \mathrm{~nm}$ long
\end{abstract}

The nuclear lamina is a filamentous meshwork 


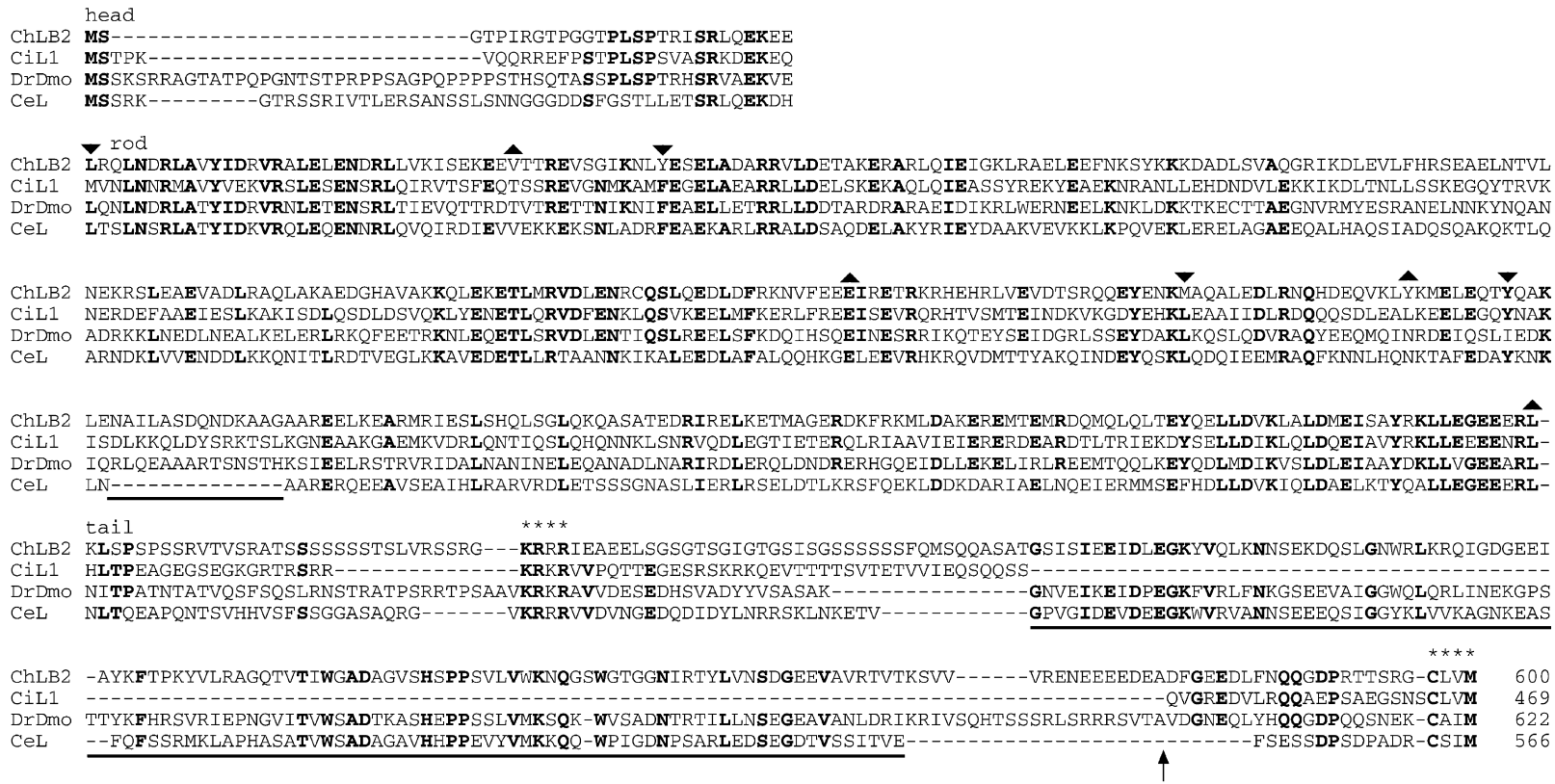

Figure 1. Sequence alignment of nuclear lamins used here. The Drosophila lamin Dmo is included as a prototype for invertebrate lamins that lack unusual sequence features such as those exhibited by the C. elegans lamin and the Ciona intestinalis lamin L1. The tripartite domain organisation (head, rod and tail) and the subdomains of the central rod (i.e. coils $1 \mathrm{a}, 1 \mathrm{~b}, 2 \mathrm{a}$ and $2 \mathrm{~b}$ delineated by arrowheads, one pointing down and the other up) are indicated. The nuclear localization signal and the terminal CaaX motif are both marked by four asterisks. Residues identical in at least three sequences are represented in bold type. The sequences are from top to bottom: chicken lamin B2 (ChLB2; accession number P14732 ${ }^{30}$ ) C. intestinalis lamin L1 (CiL1; $\alpha$ variant; accession number AJ $271075^{10}$ ), Drosophila melanogaster lamin Dmo (DrDmo; accession number P08928 ${ }^{31}$ ), and C. elegans lamin (CeL; accession number X74027 ${ }^{13}$ ). Lamin regions that vary in length are the small head and the large tail domains. Note however the moderately conserved middle region of the tail domain (i.e. the 105-residue region ${ }^{9,10}$ ) that is present in all lamins except for Ciona lamin. Yet another length variability arises in the vertebrate A lamins (sequences not shown). They have an additional exon inserted in the tail domain (approximate position marked by the arrow pointing upwards). It is inserted past the acidic cluster found in all vertebrate lamins. ${ }^{9,10}$ The unique features of two lamins are underlined: the C. elegans lamin harbors a 14-residue deletion within the rod domain, i.e. early in coil $2 b,{ }^{13}$ and the Ciona lamin $L 1^{10}$ lacks the Ig-like 105-residue region within the tail domain (see the text).

Nuclear lamins seem restricted to metazoa, as the completed genomes of the yeast Saccharomyces cerevisiae and the plant Arabidopsis thaliana lack obvious orthologs. Some 35 lamin sequences are currently known. They range from the simplest animals such as the cnidarians (Hydra and Taelia) to various other invertebrates (Caenorhabditis elegans, Drosophila, Priapulus, several echinoderms, two urochordates and the cephalochordate Amphioxus), and to a large number of vertebrates. Alignment of these sequences defined the variable and conserved domains and identified a rare lamin species that deviates from the general theme (Figure $1^{9,10}$ ). Of variable length are the relatively small (typically 30-60 residues) head domain, the region between the C-terminal end of the rod domain and the nuclear localization signal, as well as the region between this signal and the well conserved central part of the tail, the so called 105-residue region whose atomic structure has recently been solved as an Ig-like domain for human lamin A both by X-ray crystallography ${ }^{11}$ and by NMR spectroscopy. ${ }^{12}$ Length variability continues in the rest of the tail domain. This domain also includes the additional exon (about
95 residues) inserted in the larger A-type lamins that are restricted to the vertebrates. ${ }^{9}$ Nearly all lamin sequences end with the motif CaaX. In vivo this motif is processed to a cysteine residue that, in turn, is carboxyl-methylated and carries an isoprenyl derivative in thio-ether linkage.

In contrast to the two end domains the central rod domain is nearly canonically conserved in length. Even in cnidarian lamins there is only minimal variability. The linker $\mathrm{L} 2$ between coils $1 \mathrm{~b}$ and 2a is increased by one and three residues in Hydra and Taelia lamin, respectively. ${ }^{9} \mathrm{~A}$ much larger change occurs only in the lamin rod of the nematode C.elegans, which lacks 14 residues or two heptads early in coil $2 b .^{13}$ The $C$. elegans lamin arises from the only lamin gene in the genome, ${ }^{14}$ and Northern analysis indicates a single mRNA species. ${ }^{13}$ The second unusual lamin type is represented by all four sequences known for tunicates (urochordates). One of these is provided by lamin L1 of Ciona intestinalis. The tunicate lamins lack the central 105-residue region that is relatively well conserved in sequence from Hydra to Drosophila and man. ${ }^{10}$ 


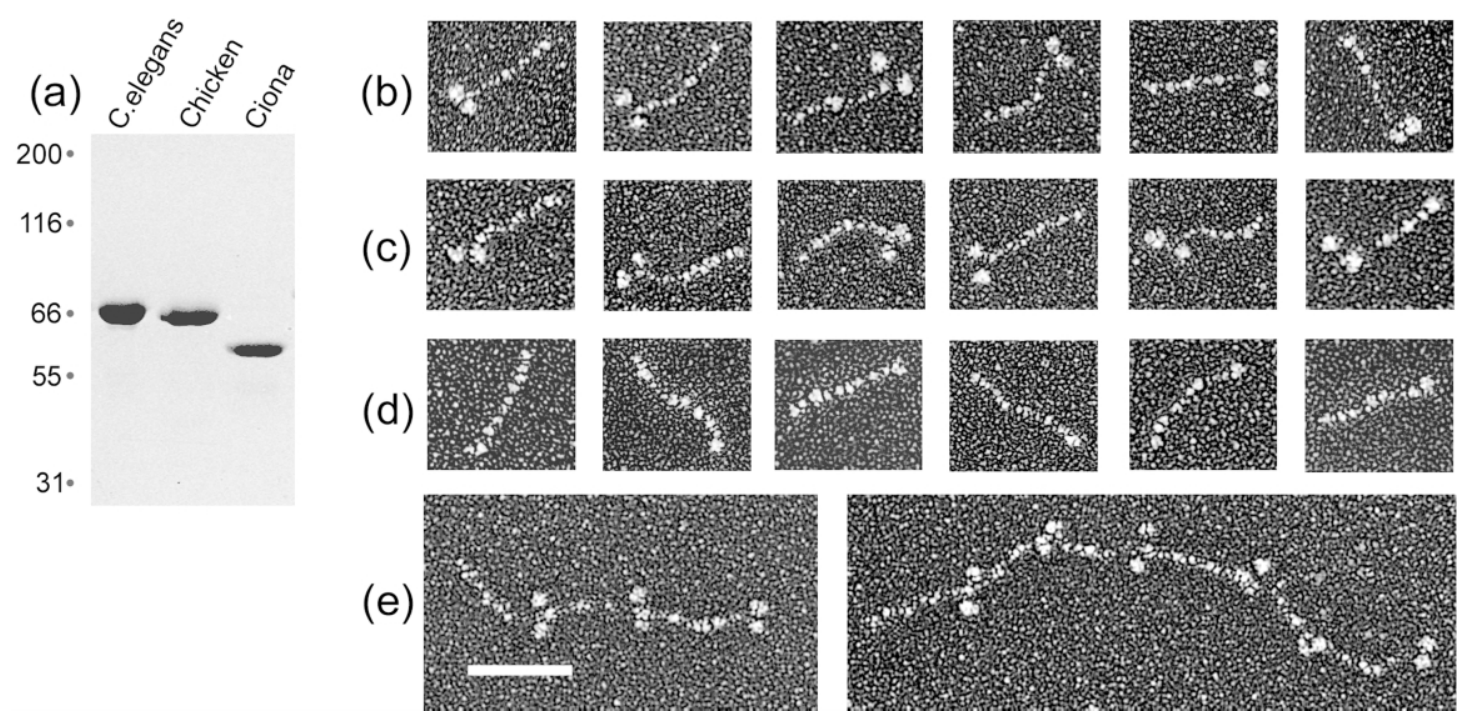

Figure 2. Purification of recombinant lamins (a), dimer formation ((b), (c) and (d)), and head-to-tail formation of dimers (e). Purification of recombinant lamins (a). The coding sequence of C. elegans lamin ${ }^{13}$ was amplified by PCR from an embryonic Uni-ZAP cDNA library (Stratagene, Heidelberg, Germany) and ligated into the expression vector pET22 (Novagen, Madison, WI, USA). E. coli BL21 (DE3) Lys S cells containing the expression plasmid were grown at $37^{\circ} \mathrm{C}$ in $200 \mathrm{ml} \mathrm{SOB}$ medium ${ }^{32}$ with $50 \mu \mathrm{g} / \mathrm{ml}$ ampicilin. Expression was induced with $1 \mathrm{mM}$ IPTG in late exponential phase. After three to four hours the cells were pelleted, washed and resuspended in guanidinium lysis buffer (6 M guanidine hydrochloride, $20 \mathrm{mM}$ sodium phosphate, $500 \mathrm{mM} \mathrm{NaCl}, \mathrm{pH} 7.8$ ) containing a protease inhibitor cocktail (Roche, Mannheim, Germany). After incubation at room temperature for ten minutes, sonication and centrifugation at $10,000 \mathrm{~g}$ the supernatant was applied onto a ProBond ${ }^{\mathrm{TM}}$ column equilibrated with denaturing buffer ( $8 \mathrm{M}$ urea, $20 \mathrm{mM}$ sodium phosphate, $500 \mathrm{mM} \mathrm{NaCl}, \mathrm{pH}$ 7.8). The eluate obtained by the same buffer at pH 6 that was highly enriched in recombinant C. elegans lamin, was applied onto a fast desalting 26/10 column (Amersham Pharmacia, Uppsala, Sweden) that was equilibrated in $8.5 \mathrm{M}$ urea containing $10 \mathrm{mM} \mathrm{Na} \mathrm{HPO}_{4}, 1 \mathrm{mM}$ 2-mercaptoethanol (pH 6.6). The protein fraction obtained was subjected to ion exchange chromatography on Mono $S$ equilibrated in the same buffer. Fractions enriched in lamin were pooled, passed through a fast desalting column in $8.5 \mathrm{M}$ urea containing $10 \mathrm{mM}$ Tris- $\mathrm{HCl}, 1 \mathrm{mM}$ 2-mercaptoethanol ( $\mathrm{pH}$ 8) and subjected to ion exchange chromatography on Mono Q equilibrated in the same buffer. Fractions containing pure C. elegans lamin were identified by SDS-PAGE. The coding sequence of chicken lamin B2 cDNA was amplified by PCR from the vector pAR3038 ${ }^{8}$ and subcloned into the bacterial expression vector pET22 (Novagen) to yield a lamin B2 with a carboxy-terminal six-histidine tag. Expression and purification of the tagged recombinant lamin B2 using ProBond ${ }^{\mathrm{TM}}$ resin was as above with the following modification. Before eluting with the denaturing buffer at $\mathrm{pH} 4$ the column was washed with the same buffer at $\mathrm{pH} 6$ and $\mathrm{pH} 5.3$. The $\mathrm{pH} 4$ protein fraction, enriched in recombinant chicken lamin B2, was subjected to ion exchange chromatography on Mono S and Mono Q as described above. Fractions containing pure lamin B2 were identified by SDS-PAGE. The coding sequence of the lamin L1 ( $\alpha$ variant) cDNA from $C$. intestinalis was amplified by PCR from the pCR2.1 vector ${ }^{10}$ and ligated into the pET23 vector (Novagen). After expression in E. coli, inclusion bodies highly enriched in recombinant Ciona lamin, were solubilized in $8.5 \mathrm{M}$ urea. Further purification of Ciona lamin by ion exchange chromatography on Mono $\mathrm{S}$ and Mono $\mathrm{Q}$ provided a pure preparation as assayed by SDS-PAGE. All purified lamins were stored in aliquots in $8.5 \mathrm{M}$ urea Tris- $\mathrm{HCl}$ buffer at $-20^{\circ} \mathrm{C}$. Lamin dimer formation ((b)-(d)). Aliquots of C. elegans, chicken and Ciona lamins in urea buffer were dialyzed for two hours against $150 \mathrm{mM} \mathrm{NaCl}, 1 \mathrm{mM}$ EGTA in $25 \mathrm{mM}$ Tris- $\mathrm{HCl}$ $(\mathrm{pH} 8.5)$ at protein concentrations of $0.4 \mathrm{mg} / \mathrm{ml}, 0.5 \mathrm{mg} / \mathrm{ml}$ and $0.25 \mathrm{mg} / \mathrm{ml}$, respectively. Dialysis was at room temperature using floating filters (mixed cellulose ester; $0.01 \mu \mathrm{m}$ pore size; Millipore, Eschborn, Germany). All buffers were $1 \mathrm{mM}$ in DTT and were made in Milli-Q water (Milli-Q standard system in organex configuration; Millipore). For glycerol spraying/rotary metal shadowing $30 \mu \mathrm{l}$ aliquots of dialyzed protein were mixed with glycerol to a final concentration of $30 \%$ and sprayed onto freshly cleaved mica. The mica was placed on the rotary table of a Balzers apparatus (BAE 120T; Balzers Pfeiffer GmbH, Asslar, Germany) and dried at room temperature in vacuo. Dried samples were rotary shadowed with platinum/carbon at an elevation angle of $3.5^{\circ}$ and coated with a carbon film..$^{33,34}$ Specimens were observed using a Philips CM12 transmission electron microscope (Philips, Eindhoven, The Netherlands) operated at $80 \mathrm{kV}$. Electron micrographs were digitised with an Agfa Duoscan T2500 scanner at a resolution of 1200 dpi. Digitised micrographs were processed using Adobe Photoshop software. Lamin dimers of C. elegans (b), chicken B2 (c) and Ciona $(\mathrm{d})$. Note the absence of the two globules at one end of the rod in the case of Ciona lamin dimers. Longitudinal head-to-tail polymers of C. elegans lamin dimers (e). Lamin in urea buffer was dialyzed for one hour against $50 \mathrm{mM} \mathrm{NaCl}, 1 \mathrm{mM}$ DTT, $50 \mathrm{mM}$ Tris- $\mathrm{HCl}(\mathrm{pH}$ 8.0) at room temperature. Glycerol spraying/rotary metal shadowing was as above. Scale bar represents $50 \mathrm{~nm}$.

Using recombinant proteins purified from Escherichia coli we have analyzed the in vitro assembly of the two unusual lamins by electron microscopy. For comparison, chicken lamin B2 served as a representative of normal lamins. The three lamins were expressed in E. coli and the proteins were purified in urea buffers. Figure 2(a) documents the purity of the preparations. Lamins 

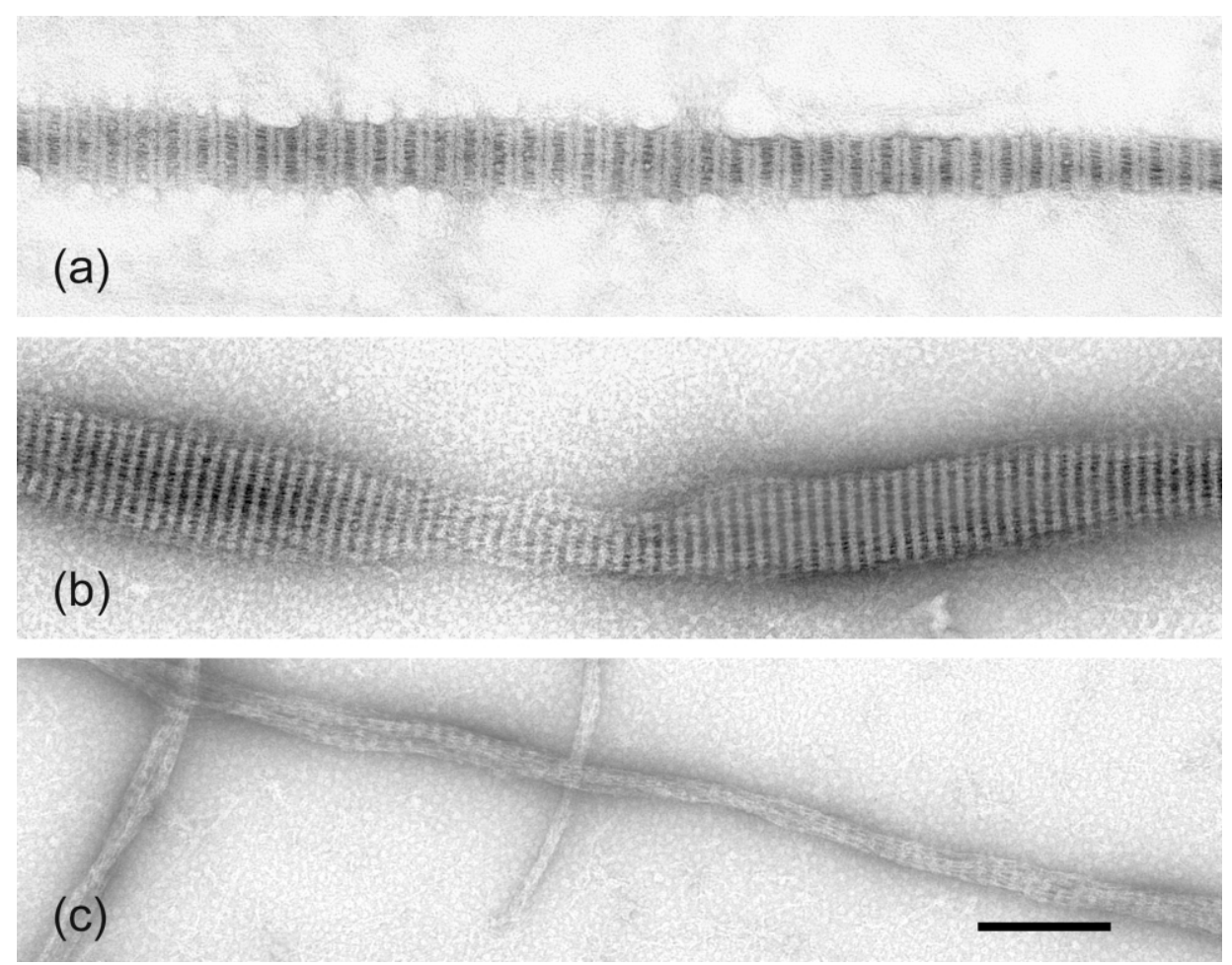

Figure 3. Lamin paracrystal formation. (a) C. elegans lamin at $0.4 \mathrm{mg} / \mathrm{ml}$ in urea buffer was dialyzed for one hour against $15 \mathrm{mM}$ Tris- $\mathrm{HCl}(\mathrm{pH} 7.4)$ followed by a two hour dialysis against $25 \mathrm{mM}$ Tris- $\mathrm{HCl}(\mathrm{pH} 9.0), 20 \mathrm{mM} \mathrm{CaCl}_{2}$. (b) Chicken lamin B2 at $0.2 \mathrm{mg} / \mathrm{ml}$ was first dialyzed against $15 \mathrm{mM}$ Tris- $\mathrm{HCl}(\mathrm{pH} \mathrm{7.4)}$ for 20 hours and then for five hours against $25 \mathrm{mM}$ Tris $-\mathrm{HCl}(\mathrm{pH} 9.0), 25 \mathrm{mM} \mathrm{CaCl}_{2}$. (c) Ciona lamin at $0.2 \mathrm{mg} / \mathrm{ml}$ was dialyzed against $20 \mathrm{mM}$ Tris- $\mathrm{HCl}(\mathrm{pH} \mathrm{8.0)}, 50 \mathrm{mM} \mathrm{NaCl}$ for one hour and then for four hours against $20 \mathrm{mM}$ Tris- $\mathrm{HCl}(\mathrm{pH} 7.2), 20 \mathrm{mM}$ $\mathrm{CaCl}_{2}$. All buffers were $1 \mathrm{mM}$ in DTT. Negative staining was with $2 \%(\mathrm{w} / \mathrm{v})$ uranyl acetate. Note the different banding patterns of the C. elegans and chicken B2 lamin paracrystals (for details see the text). Scale bar represents $200 \mathrm{~nm}$.

in $8 \mathrm{M}$ urea at $0.25-0.5 \mathrm{mg} / \mathrm{ml}$ were briefly dialyzed against $150 \mathrm{mM} \mathrm{NaCl}, 1 \mathrm{mM}$ DTT, $25 \mathrm{mM}$ Tris $-\mathrm{HCl}$ ( $\mathrm{pH}$ 8.5), and processed for glycerol spraying/rotary metal shadowing. Figure 2(b) shows that dimers of C. elegans lamin have the same ultrastructural appearance as chicken lamin B2 dimers (Figure 2(c)), which were extensively studied in the past. ${ }^{6,8}$ Both molecules display a rod with two distinct globules situated at one end. Evidently, these globules represent a distinct part of the C-terminal tail domain of these lamins. ${ }^{2,6,15}$ In fact, the Ig-like 105-residue region ${ }^{11,12}$ of human lamin A (i.e. residues $434-$ 551), when expressed in E.coli, appears after glycerol spraying/rotary metal shadowing as globules indistinguishable in size and shape from those depicted in the intact lamin dimers (K. Bechert, our unpublished results). In line with this view are the images of the Ciona lamin dimer, which misses the 105-residue region in its tail domain (Figure 1) and hence lacks the two pronounced globules at one end of its rod domain (Figure 2(d)). Measurements on 100 molecules show that the rod domain of chicken lamin $(54.2( \pm 1.2) \mathrm{nm})$ is slightly longer than the rod domain of C. elegans lamin $(52.7( \pm 1.4) \mathrm{nm})$. This $1.5-\mathrm{nm}$ length difference is in the range predicted for a 14-residue deletion (Figure 1) in coiled-coil conformation (calculated value $2 \mathrm{~nm}$ ). As described for rat, chicken and Drosophila lamin dimers $^{1,7,8}$ the C. elegans lamin dimers also formed pronounced head-to-tail polymers when dialyzed for one hour against $50 \mathrm{mM} \mathrm{NaCl}, 1 \mathrm{mM} \mathrm{DTT}$, $50 \mathrm{mM}$ Tris- $\mathrm{HCl}$ (pH 8.0) (Figure 2(e)).

To see whether the two-heptad deletion in the central rod domain of C.elegans lamin also influences the higher order assembly states we turned to low salt buffers containing 20-30 mM $\mathrm{CaCl}_{2}$, conditions that are known to favor paracrystal formation by various lamins and their derivatives. ${ }^{6-8,16}$ Figure 3 documents that in the presence of calcium ions all three lamins form paracrystals. Interestingly, the paracrystals of C. elegans and chicken B2 lamin clearly differ in their banding pattern and axial repeats. C. elegans lamin paracrystals show a broad dark band followed by two light bands that are separated by a thin dark band (Figure 3(a)). The axial repeat length obtained from 100 measurements is $44.7( \pm 0.2) \mathrm{nm}$. The unevenly spaced banding pattern of the C.elegans lamin paracrystals resembles that obtained from tailless lamins, ${ }^{2}$ and in particular from tailless chicken lamin $B 2^{6}$ and tailless Drosophila lamin Dmo. ${ }^{7}$ In contrast (Figure 3(b)), chicken lamin B2 provided the earlier reported banding pattern of alternating dark and light bands with a $\sim 24-\mathrm{nm}$ quasi-axial repeat. ${ }^{8}$ The true axial repeat includes two dark and two 

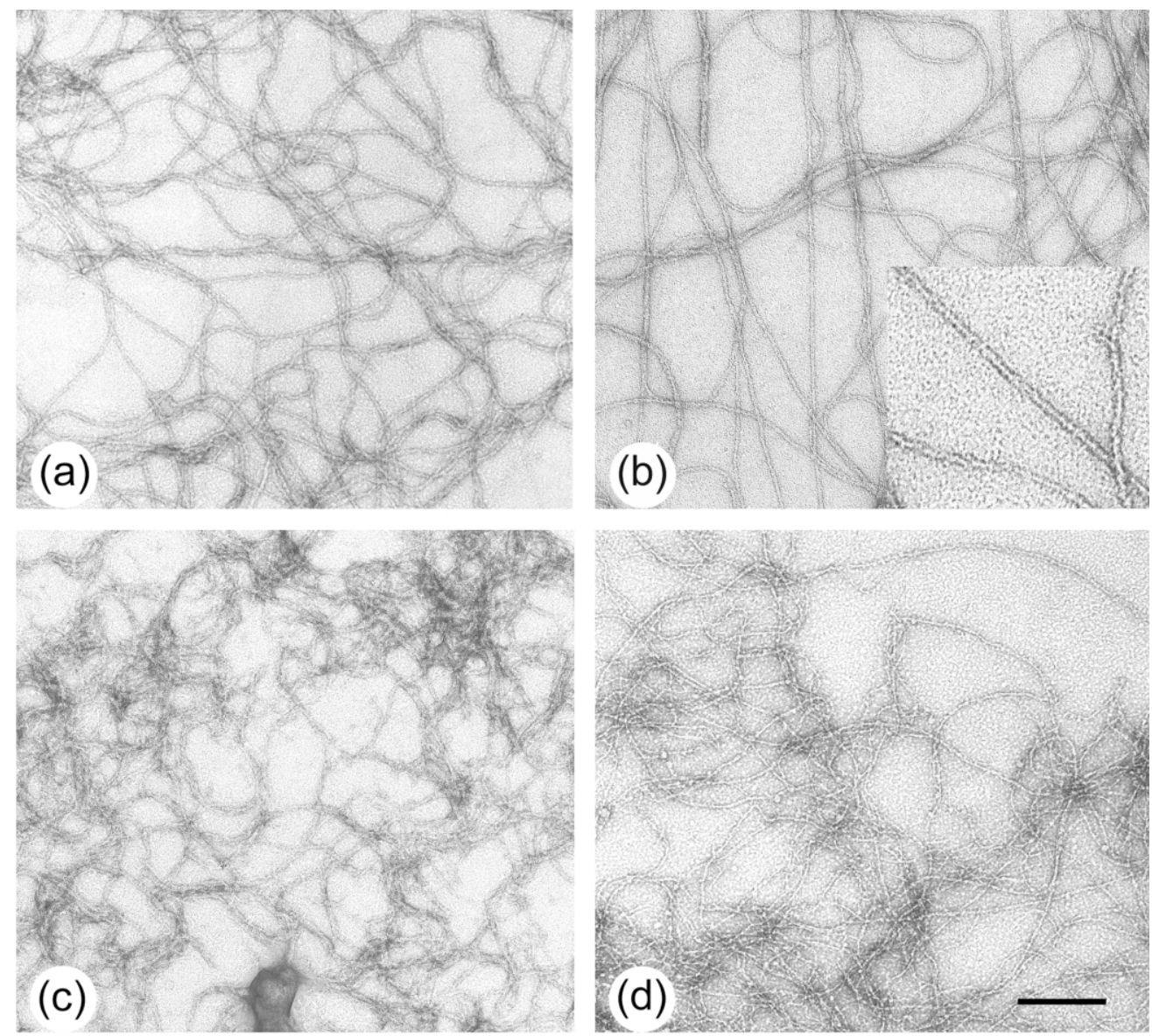

Figure 4. Lamin filament formation. ((a) and (b)) C. legans lamin $(0.1 \mathrm{mg} / \mathrm{ml})$ in urea buffer was dialyzed for four hours against $2 \mathrm{mM}$ Tris $-\mathrm{HCl}(\mathrm{pH}$ ) $)$ and then for up to 16 hours against $15 \mathrm{mM}$ Tris- $\mathrm{HCl}(\mathrm{pH} 7.4)$. This buffer was made from a $1 \mathrm{M}$ stock solution with Milli $\mathrm{Q}$ water (a). Alternatively, a $0.05 \mathrm{M}$ Tris- $\mathrm{HCl}$ buffer was made from solid Tris $-\mathrm{HCl}$ and Tris-Base according to the Sigma technical bulletin No. 106B and diluted to $15 \mathrm{mM}$ with Milli Q water (b). The inset in (b) reveals a higher magnification view of the 10-nm filaments formed by the C. elegans lamin. (c) Chicken lamin B2 $(0.15 \mathrm{mg} / \mathrm{ml})$ was dialyzed for 20 hours against $15 \mathrm{mM}$ Tris $-\mathrm{HCl}(\mathrm{pH} 7.4)$. (d) Ciona lamin $(0.2 \mathrm{mg} / \mathrm{ml})$ was dialyzed for four hours against $20 \mathrm{mM}$ Tris $-\mathrm{HCl}(\mathrm{pH}$ 8) containing $50 \mathrm{mM} \mathrm{NaCl}$. All buffers were $1 \mathrm{mM}$ in DTT. All samples were negatively stained with $2 \%$ uranyl acetate. Note the morphologically normal looking 10-nm intermediate filaments (IF) formed by the C. elegans lamin ((a) and (b)). While the chicken lamin B2 filaments yield an average diameter of $9.6 \mathrm{~nm}$, they appear much more polymorphic and exhibit a strong tendency to form tangles (c). In contrast, the Ciona lamin filaments yield an average diameter of only $5.4 \mathrm{~nm}$ (d). For details see the text. Scale bar represents $200 \mathrm{~nm}$ in (a)-(d) and $66 \mathrm{~nm}$ in the inset.

light bands and amounts to $47.8( \pm 0.4) \mathrm{nm}(100$ measurements). The 3.1-nm shorter axial repeat in the case of C. elegans (Figure 3(a)) is most likely due to the deletion of two heptads from the coiled-coil rod domain of C. elegans lamin (Figure 1 ) and is in line with the slightly reduced length of the lamin dimer molecules (Figure 2), i.e. $52.7 \mathrm{~nm}$ versus $54.2 \mathrm{~nm}$ (see above). Similarly, the different spacings of the transverse bands within the axial repeat of C.elegans versus chicken B2 lamin paracrystals may, at least in part, arise too from the missing two heptads in coil 2 of C. elegans lamin, as this yields a different stagger between antiparallel dimers that interact via their coil 2 (i.e. the A22 dimer-dimer interaction ${ }^{17}$ ). In addition, and possibly as a consequence of its shorter coil 2, the axial positioning of the globular tail domain in the C. elegans lamin paracrystals may be distinct from that in chicken lamin B2 paracrystals.
The paracrystals formed by Ciona lamin appeared much poorer in quality than those obtained from the other two lamins (Figure 3(c); compare with Figure 3(a) and (b)). Nevertheless, in many regions a distinct banding pattern could be observed and resembled that of chicken lamin B2 both in terms of appearance and axial repeat length. Since previous studies with truncated lamins lacking the entire tail domain have documented that they retain the ability to form paracrystals, $, 0,7,15,16,18-20$ we have not tried to improve the quality of the paracrystals formed by the Ciona lamin that lacks the central part (i.e. the 105-residue globule) of the tail domain (see Figure 1).

Previous studies on the in vitro assembly of chicken, human, rat and Drosophila lamins ${ }^{1,6-8,15}$ emphasized that intermediate filament-like structures (i.e. exhibiting a $\sim 10$-nm average diameter) 
are only transiently observed and absent at steady state where thick filament bundles, aggregates and paracrystalline arrays predominate. To see whether assembly conditions can be established in which C. elegans lamin forms stable IFs, we employed various low ionic strength buffers that are known to yield $10-\mathrm{nm}$ filaments with invertebrate cytoplasmic IF proteins ${ }^{21-23}$ and with mammalian keratins. $^{24-27}$ The best results were achieved when C. elegans lamin (at $0.1 \mathrm{mg} / \mathrm{ml}$ ) was dialyzed against $15 \mathrm{mM}$ Tris- $\mathrm{HCl}$ (pH 7.4). Figure 4(a) and (b) reveals IFs with an average diameter of $10.2( \pm 0.7) \mathrm{nm}$ (Figure 4(b), inset) that were obtained under these conditions in two separate experiments. Filament bundling was only rarely observed and no paracrystals were seen.

Although these low ionic strength buffers also yielded filaments with chicken B2 and Ciona lamins (Figure 4(c) and (d)), the resulting filament populations appear much more polymorphic. Chicken lamin B2, for example, formed filaments with an average diameter of $9.6( \pm 2.7) \mathrm{nm}$, i.e. very close to that of bona fide IFs. While these filaments exhibited a strong tendency to form tangles (Figure 4(c)), no paracrystals were detected under these assembly conditions. The Ciona filaments, in contrast, yielded a markedly smaller average diameter, i.e. $5.4( \pm 1.1) \mathrm{nm}$ (Figure $4(\mathrm{~d})$ ), than is common for bona fide IFs.

Here we have documented that the single nuclear lamin of the nematode C. elegans ${ }^{14}$ assembles in low ionic strength Tris- $\mathrm{HCl}$ buffer into stable 10-nm filaments that exhibit a normal IF morphology (see Figure 4(b)). This result is in line with the finding that the primary sequences of nuclear lamins and cytoplasmic IF proteins are related $^{28,29}$ and that at least in Xenopus oocytes the nuclear lamina is built as a near tetragonal lattice of 10-nm filaments. ${ }^{1}$ Nevertheless previous studies using different buffer conditions found that lamin assembly notoriously leads to thick bundles, tangles and paracrystalline arrays and that 10-nmlike filaments are practically absent at steady state (for references see above). However the organisation of the nuclear lamina in somatic metazoan cell types is not known. Thus it remains to be seen whether such laminae are also built from a sizable amount of bona fide $10-\mathrm{nm}$ lamin filaments, or whether they are formed by thinner filaments such as those that form during early in vitro assembly stages by head-to-tail polymerization of lamin dimers or several such filaments after lateral association. ${ }^{8}$

\section{Acknowledgements}

We thank Uwe Plessmann for protein sequencing. We thank Dr Erich Nigg, Munich, for providing the chicken B2 cDNA and Dr Georg Krohne, Würzburg, for a gift of recombinant Drosophila lamin Dmo. This work was supported in part by a grant from the German-Israeli Foundation to K.W., by a grant from the Swiss National Science Foundation to U.A., and by the Maurice E. Müller Foundation of Switzerland.

\section{References}

1. Aebi, U., Cohn, J., Buhle, L. \& Gerace, L. (1986). The nuclear lamina is a meshwork of intermediate-type filaments. Nature, 323, 560-564.

2. Stuurman, N., Heins, S. \& Aebi, U. (1998). Nuclear lamins: their structure, assembly, and interactions. J. Struct. Biol. 122, 42-66.

3. Hutchison, C. J., Alvarez-Reyes, M. \& Vaughn, O. A. (2001). Lamins in disease: why do ubiquitously expressed nuclear envelope proteins give rise to tissue-specific disease phenotypes? J. Cell Sci. 114, 9-19.

4. Fuchs, E. \& Weber, K. (1994). Intermediate filaments: Structure, dynamics, function and disease. Annu. Rev. Biochem. 63, 345-382.

5. Parry, D. A. D. \& Steinert, P. M. (1995). Intermediate Filament Structure, Springer, New York.

6. Heitlinger, E., Peter, M., Lustig, A., Villiger, W., Nigg, E. A. \& Aebi, U. (1992). The role of the head and tail domain in lamin structure and assembly: analysis of bacterially expressed chicken lamin A and truncated B2 lamins. J. Struct. Biol. 108, 74-89.

7. Sasse, B., Lustig, A., Aebi, U. \& Stuurman, N. (1997). In vitro assembly of Drosophila lamin Dmo; lamin polymerization properties are conserved. Eur. J. Biochem. 250, 30-38.

8. Heitlinger, E., Peter, M., Haner, M., Lustig, A., Aebi, U. \& Nigg, E. A. (1991). Expression of chicken lamin B2 in Escherichia coli: characterization of its structure, assembly, and molecular interactions. J. Cell Biol. 113, 485-495.

9. Erber, A., Riemer, D., Hofemeister, H., Bovenschulte, M., Stick, R., Panopoulou, G. et al. (1999). Characterization of the Hydra lamin and its gene; a molecular phylogeny of metazoan lamins. J. Mol. Evol. 49, 260-271.

10. Riemer, D., Wang, J., Zimek, A., Swalla, B. J. \& Weber, K. (2000). Tunicates have unusual nuclear lamins with a large deletion in the carboxyterminal tail domain. Gene, 255, 317-325.

11. Dhe-Paganon, S., Werner, E. D., Chi, Y. I. \& Shoelson, S. E. (2002). Structure of the globular tail of nuclear lamin. J. Biol. Chem. 277, 17381-17384.

12. Krimm, I., Ostlund, C., Gilquin, B., Couprie, J., Hossenlopp, P., Mornon, J. P. et al. (2002). The Ig-like structure of the C-terminal domain of lamin A/C, mutated in muscular dystrophies, cardiomyopathy, and partial lipodystrophy. Structure, 10, 811-823.

13. Riemer, D., Dodemont, H. \& Weber, K. (1993). A nuclear lamin of the nematode Caenorhabditis elegans with unusual structural features; cDNA cloning and gene organisation. Eur. J. Cell Biol. 62, 214-223.

14. Liu, J., Ben-Shahar, T. R., Riemer, D., Treinin, M., Spann, P., Weber, K. et al. (2000). Essential roles for Caenorhabditis elegans lamin gene in nuclear organization, cell cycle progression, and spatial organization of nuclear pore complexes. Mol. Biol. Cell, 11, 3937-3947. 
15. Moir, R. D., Quinlan, R. A. \& Stewart, M. (1990). Expression and characterization of human lamin C. FEBS Letters, 268, 301-305.

16. Sasse, B., Aebi, U. \& Stuurman, N. (1998). A tailless Drosophila lamin Dmo fragment reveals lateral associations of dimers. J. Struct. Biol. 123, 56-66.

17. Herrmann, H. \& Aebi, U. (1998). Intermediate filament assembly: fibrillogenesis is driven by decisive dimer-dimer interactions. Curr. Opin. Struct. Biol. 8, 177-185.

18. Moir, R. D., Donaldson, A. D. \& Stewart, M. (1991). Expression in Escherichia coli of human lamins A and $\mathrm{C}$ : influence of head and tail domains on assembly properties and paracrystal formation. J. Cell Sci. 99, 363-372.

19. Glass, C. G., Glass, J. R., Taniura, H., Hasel, K. W., Blevitt, M. \& Gerace, L. (1993). The $\alpha$-helical rod domain of human lamins $\mathrm{A}$ and $\mathrm{C}$ contains a chromatin binding site. EMBO J. 12, 4413-4424.

20. Klapper, M., Exner, K., Kempf, A., Gehrig, C., Stuurman, N., Fisher, P. A. \& Krohne, G. (1997). Assembly of A- and B-type lamins studied in vivo with the baculovirus system. J. Cell Sci. 110, 2519-2532.

21. Karabinos, A., Riemer, D., Erber, A. \& Weber, K. (1998). Homologues of vertebrate type I, II and III intermediate filament (IF) proteins in an invertebrate: the IF multigene family of the cephalochordate Branchiostoma. FEBS Letters, 437, 15-18.

22. Karabinos, A., Riemer, D., Panopoulou, G., Lehrach, H. \& Weber, K. (2000). Characterisation and tissuespecific expression of the two keratin subfamilies of intermediate filament proteins in the cephalochordate Branchiostoma. Eur. J. Cell Biol. 79, 17-26.

23. Wang, J., Karabinos, A., Schünemann, J., Riemer, D. \& Weber, K. (2000). The epidermal intermediate filament proteins of tunicates are distant keratins; a polymerisation-competent hetero coiled coil of the Styela D protein and Xenopus keratin 8. Eur. J. Cell Biol. 79, 478-487.

24. Franke, W. W., Schiller, D. L. \& Grund, C. (1982). Protofilamentous and annular structures as intermediates during reconstitution of cytokeratin filaments in vitro. Biol. Cell, 46, 257-268.
25. Aebi, U., Fowler, W. E., Rew, P. \& Sun, T. T. (1983). The fibrillar substructure of keratin filaments unraveled. J. Cell Biol. 97, 1131-1143.

26. Hatzfeld, M. \& Weber, K. (1990). The coiled coil of in vitro assembled keratin filaments is a heterodimer of type I and II keratins: use of site-specific mutagenesis and recombinant protein expression. J. Cell Biol. 110, 1199-1210.

27. Coulombe, P. A. \& Fuchs, E. (1990). Elucidating the early stages of keratin filament assembly. J. Cell Biol. 111, 153-169.

28. Fisher, D. Z., Chaudhary, N. \& Blobel, G. (1986). cDNA sequencing of nuclear lamins $A$ and $C$ reveals primary and secondary structural homology to intermediate filament proteins. Proc. Natl Acad. Sci. USA, 83, 6450-6454.

29. McKeon, F. D., Kirschner, M. W. \& Caput, D. (1986). Homologies in both primary and secondary structure between nuclear envelope and intermediate filament proteins. Nature, 319, 463-468.

30. Vorburger, K., Lehner, C. F., Kitten, G. T., Eppenberger, H. M. \& Nigg, E. A. (1989). A second higher vertebrate B-type lamin. cDNA sequence determination and in vitro processing of chicken lamin B2. J. Mol. Biol. 208, 405-415.

31. Gruenbaum, Y., Landesman, Y., Drees, B., Bare, J. W., Saumweber, H., Paddy, M. R. et al. (1988). Drosophila nuclear lamin precursor Dmo is translated from either of two developmentally regulated mRNA species apparently encoded by a single gene. J. Cell Biol. 106, 585-596.

32. Sambrook, J., Fritsch, E. F. \& Maniatis, T. (1989). Molecular Cloning: A Laboratory Manual, 2nd edit., Cold Spring Harbor Laboratory Press, Cold Spring Harbor, NY.

33. Fowler, W. E. \& Aebi, U. (1983). Preparation of single molecules and supramolecular complexes for highresolution metal shadowing. J. Ultrastruct. Res. 83, 319-334.

34. Häner, M., Bremer, A., Aebi, U., et al. (1997). Glycerol spraying/rotary metal shadowing. In Cell Biology; A Laboratory Hand Book (Celis, J. E., ed.), 2nd edit., vol. 3, pp. 292-298, Academic Press, New York.

Edited by W. Baumeister 